\title{
Modelling Reflection and Transmission of Acoustic Waves at a Semiconductor: Fluid Interface
}

\author{
A. Sharma, J. N. Sharma, and Y. D. Sharma \\ Department of Mathematics, National Institute of Technology, Hamirpur 177005, India \\ Correspondence should be addressed to A. Sharma, amit.sharma3178@gmail.com
}

Received 25 May 2011; Revised 30 October 2011; Accepted 17 December 2011

Academic Editor: Joseph CS Lai

Copyright () 2012 A. Sharma et al. This is an open access article distributed under the Creative Commons Attribution License, which permits unrestricted use, distribution, and reproduction in any medium, provided the original work is properly cited.

The paper concentrates on the study of reflection and transmission characteristics of acoustic waves at the interface of a semiconductor half-space underlying an inviscid liquid. The reflection and transmission coefficients varying with the incident angles are examined. Calculated results are verified by considering the quasilongitudinal $(q P)$ and quasitransverse $(q S V)$ waves. The special cases of normal and grazing incidence are also derived and discussed. Finally, the numerical computations of reflection and transmission coefficients are carried out with the help of Gauss elimination method by using MATLAB programming software for silicon $(\mathrm{Si})$ and germanium $(\mathrm{Ge})$ semiconductors. The computer simulated-results have been plotted graphically for $\mathrm{Si}$ and presented in tabular form in case of Ge semiconductors. The study may be useful in semiconductors, geology, and seismology in addition to surface acoustic wave (SAW) devices.

\section{Introduction}

Jeffrey's [1] and Gutenberg [2] considered the reflection of elastic plane waves at the surface of a solid halfspace. Sidhu and Singh [3] investigated the propagation of plane waves in a prestressed elastic solid possessing orthotropic symmetry and showed that the velocities of $q L$ and $q S V$ waves depend upon the angle of propagation. Rayleigh [4] considered the reflection and transmission of waves from an undulated boundary surface of an elastic solid. Knott [5] derived the equations for reflection and refraction of waves at plane boundaries. The reflection and refraction phenomenon of elastic waves in solids under different situations has been treated in detail as reported in books [6-8]. Deresiewicz [9] studied the reflection of a plane waves from the stressfree boundaries of thermoelastic halfspace. Abo-dahab [10] studied the propagation of $\mathrm{P}$ waves from the stress-free surface of elastic half-space with voids under the influence of thermal relaxation and magnetic field. It is found that the angle of incidence $(\theta)$ significantly affects the reflection coefficients and the thermal relaxation time has negligible small effect on the amplitude of reflection coefficients. Madeo and Gavrilyuk [11] studied the propagation of acoustic waves in porous media including their reflection and transmission at pure fluid/porous medium permeable interface. A. N. Sinha and S. B. Sinha [12] studied the reflection of generalized thermoelastic waves from the free surface of a solid halfspace. Sharma et al. [13] studied the reflection of generalized thermoelastic waves from the boundary of a transversely isotropic halfspace. Abd-alla [14] considered the effect of relaxation time on reflection of generalized magnetothermoelastic waves. Lockett [15] studied the effect of thermal properties of a solid on the velocity of waves. Chadwick and Snedon [16] studied the reflection of plane waves in an elastic solid conducting heat.

Maruszewski [17] presented theoretical considerations of the simultaneous interactions of elastic, thermal, and diffusion of charge carrier fields in order to study surface waves in semiconductors. Sharma and Thakur [18] nondimensionalized the model [17] and studied the plane harmonic elastodiffusive surface wave in semiconductor material. Recently, J. N. Sharma and A. Sharma [19] studied the reflection of acoustodiffusive waves from the stress-free boundary of a semiconductor halfspace. As per knowledge of authors no study of reflection and transmission of waves based on the models of basic governing equations 


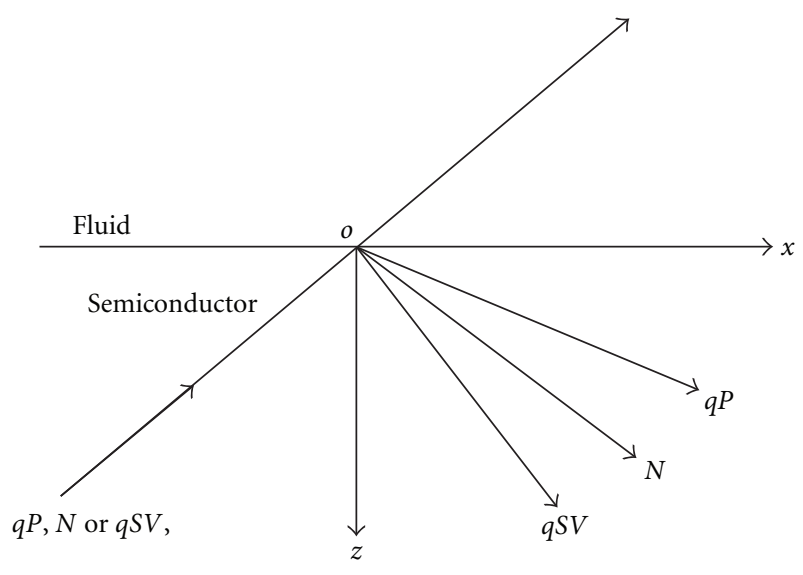

FIGURE 1: Geometry of the problem.

given in references $[17,18]$ is available in the literature. Ultrasonic waves are reflected at boundaries due to the acoustic impedance mismatch of the materials on each side. Reflection and transmission coefficients are utilized for the conversion of longitudinal to shear waves and vice versa. This feature is extremely useful in the construction of shear wave transducers. Reflection coefficients also affect the response of a transducer to a sinusoidal signal and are useful in sonography as well as in signal processing.

Keeping in view the above and applications of semiconductors in acoustic devices, the present paper is an attempt to explore the reflection and transmission characteristics of elastic waves at the interface between elastic semiconductor (n or p-type) halfspace and inviscid liquid semispace. The mathematical model consisting of governing partial differential equations of motion and charge carriers' diffusion of n-type and p-type semiconductors has been solved both analytically and numerically in the study. The computer-simulated results so obtained with the help of MATLAB programming in respect of in case of silicon ( $\mathrm{Si}$ ) and germanium $(\mathrm{Ge})$ semiconductors in contact with water have been illustrated graphically.

\section{Formulation of the Problem}

We take the origin of rectangular Cartesian coordinate system $o x y z$ at a fixed point on the boundary of the semiconductor halfspace with positive $z$-axis directed normally into the solid medium and $x$-axis along the direction of propagation of waves; the $y$-axis is taken in the direction of the line of intersection of the plane wave front with the plane surface as shown in Figure 1. If we restrict our analysis to plain strain in the $x z$-plane, all the field variables may be taken as function of $x, z$, and $t$ only. The basic governing equations of motion and diffusion of charge carrier fields for a homogeneous isotropic, elastic (n-type and p-type) semiconductors, in the absence of body forces and electromagnetic forces, are given as $[17,18]$. n-type semiconductor

$$
\begin{aligned}
& \mu \nabla^{2} \vec{u}+(\lambda+\mu) \nabla \nabla \cdot \vec{u}-\lambda^{n} N=\rho \ddot{\vec{u}}, \\
& \rho D^{n} \nabla^{2} N+\rho\left[\frac{1}{t_{n}^{+}}-\left(1-\frac{t^{n}}{t_{n}^{+}}\right) \frac{\partial}{\partial t}-t^{n} \frac{\partial^{2}}{\partial t^{2}}\right] N \\
& \quad-a_{2}^{n} T_{0} \lambda^{T} \nabla \cdot \dot{\vec{u}}=0,
\end{aligned}
$$

p-type semiconductor

$$
\begin{aligned}
& \mu \nabla^{2} \vec{u}+(\lambda+\mu) \nabla \nabla \cdot \vec{u}-\lambda^{p} P=\rho \ddot{\vec{u}}, \\
& \rho D^{p} \nabla^{2} P+\rho\left[\frac{1}{t_{p}^{+}}-\left(1-\frac{t^{p}}{t_{p}^{+}}\right) \frac{\partial}{\partial t}-t^{p} \frac{\partial^{2}}{\partial t^{2}}\right] P \\
& \quad-a_{2}^{p} T_{0} \lambda^{T} \nabla \cdot \dot{\vec{u}}=0,
\end{aligned}
$$

where $\nabla^{2}=\left(\partial^{2} / \partial x^{2}\right)+\left(\partial^{2} / \partial z^{2}\right)$ is the Laplacian operator, $N(x, z, t)=n-n_{0}$ is the electron concentration change, $P(x, z, t)=p-p_{0}$ is the hole concentration change, and $\vec{u}(x, z, t)=(u, 0, w)$ is the displacement vector. Here $\lambda, \mu$ are Lame parameters; $\rho$ is the density of the semiconductor; $\lambda^{n}=(3 \lambda+2 \mu) \alpha_{N}$ and $\lambda^{p}=(3 \lambda+2 \mu) \alpha_{P}$ are the elastodiffusive constants of electrons; $\alpha_{N}, \alpha_{P}$ are the coefficients of linear electron and holes concentration expansions. $D^{n}$ and $D^{p}$ are the diffusion coefficients of electron and hole carriers; $t_{n}, t_{p}$ and $t_{n}^{+}, t_{p}^{+}$are the relaxation and life times of the electron and hole charge carriers, respectively; $n, p$ and $n_{0}, p_{0}$ are, respectively, the nonequilibrium and equilibrium values of electrons and holes concentrations of the semiconductors; $T_{0}$ and $\lambda^{T}=(3 \lambda+2 \mu) \alpha_{T}$ are the uniform temperature and adiabatic thermomechanical coupling constant, respectively; $a_{2}^{n}, a_{2}^{p}$ are the flux-like parameters. The superposed dot represents differentiation with respect to time.

Further (1)-(2) are subjected to following assumptions $[17,18]$.

(i) All the considerations are made in the frame work of the phenomenological model.

(ii) The electric neutrality of the semiconductor is satisfied.

(iii) The magnetic field effect is ignored.

(iv) The mass of charge carriers is negligible.

(v) The electric field with in the boundary layer is very weak and can be neglected.

(vi) The recombination functions of electrons and holes are selected on the basis of facts that take care of the defects and hence the concentration values of the charge carrier fields.

The nonvanishing components of stress tensor in the semiconductor are given by

n-type:

$\tau_{z z}=(\lambda+2 \mu) \frac{\partial w}{\partial z}+\lambda \frac{\partial u}{\partial x}-\lambda^{n} N, \quad \tau_{x z}=\mu\left(\frac{\partial u}{\partial z}+\frac{\partial w}{\partial x}\right)$ 


$$
\begin{aligned}
& \text { p- type: } \\
& \tau_{z z}=(\lambda+2 \mu) \frac{\partial w}{\partial z}+\lambda \frac{\partial u}{\partial x}-\lambda^{p} P, \quad \tau_{x z}=\mu\left(\frac{\partial u}{\partial z}+\frac{\partial w}{\partial x}\right) .
\end{aligned}
$$

The basic governing equation for inviscid fluid medium is given by

$$
\lambda_{L} \nabla \nabla \cdot \vec{u}_{L}=\rho_{L} \ddot{\vec{u}}
$$

where $\lambda_{L}$ is bulk modulus and $\rho_{L}$ and $\vec{u}_{L}$ are the density of the fluid and velocity vector, respectively.

We define the quantities

$$
\begin{array}{cc}
x^{\prime}=\frac{\omega^{*} x}{c_{1}}, \quad z^{\prime}=\frac{\omega^{*} z}{c_{1}}, & t^{\prime}=\omega^{*} t, \\
N^{\prime}=\frac{N}{n_{0}}, \quad w^{\prime}=\frac{\rho \omega^{*} c_{1}}{\lambda^{n} n_{0}} w, & u^{\prime}=\frac{\rho \omega^{*} c_{1}}{\lambda^{n} n_{0}} u, \\
\tau_{i j}^{\prime}=\frac{\tau_{i j}}{\lambda^{n} n_{0}}, \quad t^{n^{\prime}}=t^{n} \omega^{*}, & t_{n}^{+^{\prime}}=t_{n}^{+} \omega^{*}, \\
\delta_{L}^{2}=\frac{c_{L}^{2}}{c_{1}^{2}}, \quad c_{L}^{2}=\frac{\lambda_{L}}{\rho_{L}}, & \delta^{2}=\frac{c_{2}^{2}}{c_{1}^{2}}, \\
\omega^{*}=\frac{c_{1}^{2}}{D^{n}}, \quad c_{1}^{2}=\frac{\lambda+2 \mu}{\rho}, & c_{2}^{2}=\frac{\mu}{\rho}, \\
\varepsilon_{n}=\frac{a_{2}^{n} T_{0} \lambda^{T} \lambda^{n}}{\rho(\lambda+2 \mu)}, \quad u_{L}^{\prime}=\frac{\rho \omega^{*} c_{1}}{\lambda^{n} n_{0}} u_{L},
\end{array}
$$

where $\omega^{*}$ is the elastodiffusive characteristic frequency and $c_{1}, c_{2}$ are, respectively, the longitudinal and shear wave velocities. Here $\varepsilon_{L}$ is the thermomechanical coupling and $c_{L}$ is the velocity of sound in the fluid. Such quantities in case of p-type semiconductor can be written from those in (6) by replacing the subscript/superscripts $n$ with $p$ and the quantity $N$ with $P$. Upon using quantities (6) in (1)-(4), and (5), we obtain (n-type semiconductor)

$$
\begin{gathered}
\delta^{2} \nabla^{2} \vec{u}+\left(1-\delta^{2}\right) \nabla \nabla \cdot \vec{u}-\nabla N=\ddot{\vec{u}} \\
\nabla^{2} N-\left[t^{n} \frac{\partial^{2}}{\partial t^{2}}+\left(1-\frac{t^{n}}{t_{n}^{+}}\right) \frac{\partial}{\partial t}-\frac{1}{t_{n}^{+}}\right] N-\varepsilon_{n} \nabla \cdot \dot{\vec{u}}=0 \\
\tau_{x z}=\delta^{2}\left(\frac{\partial u}{\partial z}+\frac{\partial w}{\partial x}\right), \quad \tau_{z z}=\left(1-2 \delta^{2}\right) \frac{\partial u}{\partial x}+\frac{\partial w}{\partial z}-N,
\end{gathered}
$$

$$
\delta_{L}^{2} \nabla \nabla \cdot \vec{u}_{L}=\ddot{\vec{u}}_{L}
$$

The equations for $\mathrm{p}$-type semiconductor can be written from (8) and (9) by replacing $N$ with $P$ and superscripts/subscripts $n$ with $p$.

We introduce the elastic potential functions $\phi$ and $\psi$ through the relations

$$
u=\frac{\partial \phi}{\partial x}+\frac{\partial \psi}{\partial z}, \quad w=\frac{\partial \phi}{\partial z}-\frac{\partial \psi}{\partial x} .
$$

However, in general, such a decomposition of displacement vector is not possible in case of anisotropic materials [3].
Upon introducing (11) in (8) and (9), we get

$$
\nabla^{2} \phi-\ddot{\phi}-N=0
$$

$$
\nabla^{2} N-\left[t^{n} \frac{\partial^{2}}{\partial t^{2}}+\left(1-\frac{t^{n}}{t_{n}^{+}}\right) \frac{\partial}{\partial t}-\frac{1}{t_{n}^{+}}\right] N-\varepsilon_{n} \nabla^{2} \dot{\phi}=0
$$

$$
\nabla^{2} \psi=\frac{\ddot{\psi}}{\delta^{2}} .
$$

Similarly for p-type semiconductor, we have

$$
\begin{gathered}
\nabla^{2} \phi-\ddot{\phi}-P=0, \\
\nabla^{2} P-\left[t^{p} \frac{\partial^{2}}{\partial t^{2}}+\left(1-\frac{t^{p}}{t_{p}^{+}}\right) \frac{\partial}{\partial t}-\frac{1}{t_{p}^{+}}\right] P-\varepsilon_{p} \nabla^{2} \dot{\phi}=0, \\
\nabla^{2} \psi=\frac{\ddot{\psi}}{\delta^{2}} .
\end{gathered}
$$

In case the semiconductors are of relaxation type, the life time and relaxation time become comparable to each other $\left(t^{n} \cong t_{n}^{+}\right)$, and consequently, (14) get simplified. The stresses (10) in terms of potential functions $\phi$ and $\psi$ with the help of (12) to (18) become

$\tau_{z z}=\ddot{\phi}-2 \delta^{2}(\phi, x x+\psi, x z), \quad \tau_{x z}=\ddot{\psi}+2 \delta^{2}(\phi, x z-\psi, x x)$

for both n-type and p-type semiconductors. In the fluid medium, the nondimensional displacements are related to scalar and vector velocity potential through the relations given by

$$
u_{L}=\phi_{L, X}, \quad w_{L}=\phi_{L, Z} .
$$

Substituting (20) in (12) we get

$$
\delta^{2} \nabla^{2} \phi_{L}-\ddot{\phi}_{L}=0 .
$$

This is the equation for waves in the inviscid fluid.

\section{Boundary Conditions}

Following sets of boundary conditions are assumed to hold at solid-fluid interface $z=0$ of the semiconductor halfspace [20].

(1) The magnitude of the normal component of stress tensor of the elastic half-space should be equal to the pressure of the liquid. This implies that

$$
\frac{\ddot{\phi}}{\delta^{2}}-2\left(\phi_{, x x}+\psi_{, x z}\right)=\frac{\rho_{L} \ddot{\phi}_{L}}{\rho \delta^{2}} .
$$

(2) The tangential components of stress tensor of the solid should be zero, which implies that

$$
\frac{\ddot{\psi}}{\delta^{2}}-2\left(\psi_{, x x}-\phi_{, x z}\right)=0 .
$$


(3) The normal component of displacement of the solid should be equal to that of fluid, which implies that

$$
\phi_{, z}-\psi_{, x}=\phi_{L_{, z}}
$$

(4) The electron and hole charge carrier fields satisfy the following conditions at the interface $z=0$

$$
\begin{aligned}
& \frac{\partial N}{\partial z}+h_{n}\left(1+t^{n} \frac{\partial}{\partial t}\right) N=0 \\
& \frac{\partial P}{\partial z}+h_{p}\left(1+t^{p} \frac{\partial}{\partial t}\right) P=0
\end{aligned}
$$

where $h_{n}=s^{n} / c_{1}, h_{p}=s^{p} / c_{1}, s^{n}$ and $s^{p}$ surface recombination velocities of electron and holes, respectively.

\section{Reflection and Transmission of Plane Waves}

We assume plane wave solution of the form

$$
\begin{aligned}
\left(\phi, \psi, N, P, \phi_{L}\right)= & (A, B, C, D, E) \\
& \times \exp \{\iota k(x \sin \theta-z \cos \theta-v t)\},
\end{aligned}
$$

where $v=\omega / k, \omega$ is circular frequency, and $k$ is the wave number. Upon using (26) in (13)-(18) and in (21) we obtain a system of algebraic equations in unknowns $A, B, C, D$. The condition for the existence of nontrivial solution of these systems of equations provides us

n-type:

$$
k_{1}^{2}=a_{1}^{2} \omega^{2}, \quad k_{2}^{2}=a_{2}^{2} \omega^{2}, \quad k_{3}^{2}=\frac{\omega^{2}}{\delta^{2}}, \quad k_{4}^{2}=a_{4}^{2} \omega^{2},
$$

p-type:

$$
k_{1}^{* 2}=a^{* 2} \omega^{2}, \quad k_{2}^{* 2}=a_{2}^{* 2} \omega^{2}, \quad k_{3}^{* 2}=\frac{\omega^{2}}{\delta^{2}}, \quad k_{4}^{* 2}=a_{4}^{* 2} \omega^{2},
$$

where

$$
\begin{gathered}
a_{1}^{2}+a_{2}^{2}=1+\alpha_{n}^{*}+\iota \omega^{-1} \varepsilon_{n}, \\
a_{1}^{2} a_{2}^{2}=\alpha_{n}^{*}, \quad a_{3}^{2}=\frac{1}{\delta^{2}}, \quad a_{4}^{2}=\frac{1}{\delta_{L}^{2}}, \\
a_{1}^{* 2}+a_{2}^{* 2}=1+\alpha_{p}^{*}+\iota \omega^{-1} \varepsilon_{p}, \quad a_{1}^{2} a_{2}^{2}=\alpha_{p}^{*} .
\end{gathered}
$$

\section{Reflection and Transmission in Case of $q P$ Wave Incidence}

Let the suffixes $i$ and $r$ represent incident and reflected waves, respectively. Omitting the term $\exp (-\iota \omega t)$, the solution (26) for the function $\phi, \psi, N, \phi_{L}$ and $P$ in case of incidence and reflected waves can be written as

$$
\begin{aligned}
\phi_{i} & =A_{i} \exp \left\{i k_{1}\left(x \sin \theta_{1}-z \cos \theta_{1}\right)\right\}, \\
\phi_{r} & =\sum_{j=1}^{2} A_{j} \exp \left\{i k_{j}\left(x \sin \theta_{j}+z \cos \theta_{j}\right)\right\}, \\
\psi_{r} & =A_{3} \exp \left\{i k_{3}\left(x \sin \theta_{3}+z \cos \theta_{3}\right)\right\}, \\
N_{i} & =S_{1} A_{i} \exp \left\{\iota k_{1}(x \sin \theta-z \cos \theta)\right\}, \\
N_{r} & =\sum_{j=1}^{2} S_{j} A_{j} \exp \left\{l k_{j}\left(x \sin \theta_{j}+z \cos \theta_{j}\right)\right\}, \\
P_{i} & =S_{1}^{*} A_{i} \exp \left\{\imath k_{1}^{*}(x \sin \theta-z \cos \theta)\right\}, \\
P_{r} & =\sum_{j=1}^{2} S_{j}^{*} A_{j} \exp \left\{l k_{j}^{*}\left(x \sin \theta_{j}+z \cos \theta_{j}\right)\right\},
\end{aligned}
$$

where

$$
\begin{gathered}
S_{j}=\omega^{2}-k_{j}^{2}=\omega^{2}\left(1-a_{j}^{2}\right), \quad j=1,2, \\
S_{j}^{*}=\omega^{2}-k_{j}^{* 2}=\omega^{2}\left(1-a_{j}^{* 2}\right), \quad j=1,2 .
\end{gathered}
$$

In the absence of electron field $\left(N=0=\varepsilon_{n}\right)$ and hole carriers $\left(P=0=\varepsilon_{p}\right)$, we have

$$
\begin{gathered}
\text { n-type: } a_{1}^{2}=1, a_{2}^{2}=\alpha_{n}^{*}, a_{3}^{2}=\frac{1}{\delta^{2}}, a_{4}^{2}=\frac{1}{\delta_{L}^{2}}, \\
\text { p-type: } a_{1}^{* 2}=1, a_{2}^{* 2}=\alpha_{p}^{*}, \\
S_{1}=0, \quad S_{2}=\omega^{2}\left(1-\alpha_{n}^{*}\right), \\
S_{1}^{*}=0, \quad S_{2}^{*}=\omega^{2}\left(1-\alpha_{p}^{*}\right) .
\end{gathered}
$$

Case 1 (Quasilongitudinal $(q P)$ wave incidence at an interface from the semiconductor). In this and the following sections, we shall confine our discussion to n-type semiconductor unless stated otherwise, and results in case of p-type semiconductor can be written from the expressions of various quantities obtained here by adopting the same procedure.

Because of coupling between various field functions the reflected fields in case of $q P$ wave incidence at the interface are given by

$$
\begin{aligned}
& \phi=\phi_{i}+\phi_{r}, \quad N=N_{i}+N_{r}, \quad P=P_{i}+P_{r}, \\
& \psi=\psi_{r}, \quad \phi_{L}=\phi_{L r} .
\end{aligned}
$$

Upon using the above equations, we calculate the stresses from (19) and then employing the boundary conditions (22)-(25) to obtain a system of four coupled algebraic equations given in the appendix. Since all the waves, incident, reflected, or transmitted, must be in phase at the surface $z=0$ for all values of $x$ and $t$, therefore from equations (A.1) we have

$$
k_{1} \sin \theta=k_{1} \sin \theta_{1}=k_{2} \sin \theta_{2}=k_{3} \sin \theta_{3}=k_{4} \sin \theta_{4} .
$$

Upon using (27) in the above relation, we obtain

$$
\theta=\theta_{1}, \quad a_{1} \sin \theta_{1}=a_{2} \sin \theta_{2}=\frac{1}{\delta} \sin \theta_{3}=a_{4} \sin \theta_{4} .
$$


This is modified form of the Snell's law for the considered material. In the absence of electron field $\left(N=0, \varepsilon_{n}=0\right)$, (35) becomes

$$
\delta \sin \theta_{1}=\sin \theta_{3} \Longrightarrow \frac{\sin \theta_{1}}{c_{1}}=\frac{\sin \theta_{3}}{c_{2}} .
$$

This is Snell's law [6]. Solving the systems of equations (A.2) with the help of Gauss elimination method, the amplitude ratios $R_{k}^{q P}(k=1,2,3)$ and $T_{1}^{q P}$ are obtained as

$$
\begin{aligned}
R_{1}^{q P}= & 1-\frac{2 a_{2} S_{2} \cos \theta_{2} \cos ^{2} 2 \theta_{3}}{\bar{a} \Delta_{L}-\Delta_{S}}, \\
R_{2}^{q P}= & \frac{-2 S_{1} a_{1} a_{4} \delta \cos \theta_{4} \cos \theta_{1} \cos ^{2} 2 \theta_{3}}{\bar{\rho} \Delta_{L}-a_{4} \delta \cos \theta_{4} \Delta_{S}} \\
= & \frac{-2 S_{1} a_{1} \cos \theta_{1} \cos ^{2} 2 \theta_{3}}{\bar{a} \Delta_{L}-\Delta_{S}}, \\
R_{3}^{q P}= & \frac{2 a_{1} a_{2} \delta^{2}\left(a_{1} S_{2} \sin 2 \theta_{1} \cos \theta_{2}-a_{2} S_{1} \cos \theta_{1} \sin 2 \theta_{2}\right) \cos 2 \theta_{3}}{\bar{a} \Delta_{L}-\Delta_{S}}, \\
T_{1}^{q P}= & \frac{2 a_{1} a_{2} \cos \theta_{1} \cos \theta_{2} \cos 2 \theta_{3}\left(S_{2}-S_{1}\right)}{a_{4} \cos \theta_{4}\left(\bar{a} \Delta_{L}-\Delta_{S}\right)} \\
& +\frac{a_{1} a_{2} \delta \sin 2 \theta_{3}\left(a_{1} S_{2} \cos \theta_{2} \sin 2 \theta_{1}-a_{2} S_{1} \cos \theta_{1} \sin 2 \theta_{2}\right)}{a_{4} \cos \theta_{4}\left(\bar{a} \Delta_{L}-\Delta_{S}\right)},
\end{aligned}
$$

where

$$
\begin{aligned}
\bar{a}= & \frac{\bar{\rho}}{a_{4} \delta \cos \theta_{4}}, \\
\Delta_{L}= & \cos \theta_{1} \cos \theta_{2} \cos 2 \theta_{3} a_{1} a_{2} \delta\left(S_{2}-S_{1}\right) \\
& +a_{1} a_{2} \delta^{2}\left(a_{1} S_{2} \sin 2 \theta_{1} \cos \theta_{2}-a_{2} S_{1} \sin 2 \theta_{2} \cos \theta_{1}\right) \sin \theta_{3},
\end{aligned}
$$

$\Delta_{S}=\left[a_{1} S_{1} \cos \theta_{1}\left[\cos ^{2} 2 \theta_{3}+a_{2}^{2} \delta^{2} \sin 2 \theta_{2} \sin 2 \theta_{3}\right]\right.$

$$
\left.-a_{2} S_{2} \cos \theta_{2}\left[\cos ^{2} 2 \theta_{3}+a_{1}^{2} \delta^{2} \sin 2 \theta_{1} \sin 2 \theta_{3}\right]\right] \text {. }
$$

In the absence of fluid medium (37) becomes

$$
\begin{aligned}
R_{1}^{q P}= & \frac{a_{1} S_{1} \cos \theta_{1}\left[\cos ^{2} 2 \theta_{3}+a_{2}^{2} \delta^{2} \sin 2 \theta_{2} \sin 2 \theta_{3}\right]}{\Delta_{S}} \\
& +\frac{a_{2} S_{2} \cos \theta_{2}\left[\cos ^{2} 2 \theta_{3}-a_{1}^{2} \delta^{2} \sin 2 \theta_{1} \sin 2 \theta_{3}\right]}{\Delta_{S}}, \\
R_{2}^{q P}= & \frac{-2 a_{1} S_{1} \cos ^{2} 2 \theta_{3} \cos \theta_{1}}{\Delta_{S}}, \\
R_{3}^{q P}= & \frac{2 a_{1}^{2} a_{2} \delta^{2} \cos 2 \theta_{3}\left[a_{2} S_{1} \sin 2 \theta_{2} \cos \theta_{1}-a_{1} S_{2} \cos \theta_{2} \sin 2 \theta_{1}\right]}{\Delta_{S}} .
\end{aligned}
$$

In the absence of fluid medium and electron field $(N=$ $0, \varepsilon_{n}=0$ ), the expression (37) and (42) for amplitude ratios with the help of (32) becomes

$$
\begin{gathered}
R_{1}^{q P}=\frac{\delta^{2} \sin 2 \theta_{1} \sin 2 \theta_{3}-\cos ^{2} 2 \theta_{3}}{\delta^{2} \sin 2 \theta_{1} \sin 2 \theta_{3}+\cos ^{2} 2 \theta_{3}}, \\
R_{2}^{q P}=0, \\
R_{3}^{q P}=\frac{2 \delta^{2} \sin 2 \theta_{1} \cos 2 \theta_{3}}{\delta^{2} \sin 2 \theta_{1} \sin 2 \theta_{3}+\cos ^{2} 2 \theta_{3}} .
\end{gathered}
$$

These relations are in complete agreement with the corresponding equations as given by Achenbach [6] in case of elastokinetics. In case of grazing incidence, $\left(\theta=90^{\circ}=\theta_{1}\right)$ the amplitude ratios given by (37)-(39) with the use of Snell's law provide us

$$
R_{1}^{q P}=-1, \quad R_{2}^{q P}=0, \quad R_{3}^{q P}=0 .
$$

This shows that $q S V$ wave and electron waves are not reflected and $q P$ wave annihilates itself being $180^{\circ}$ out of phase with the incident wave. Similarly for normal incidence $(\theta=$ $\left.0^{\circ}=\theta_{1}\right)$, the corresponding values of reflection coefficients from (37)-(42) are again obtained as

$$
R_{1}^{q P}=-1, \quad R_{2}^{q P}=0, \quad R_{3}^{q P}=0=T_{1}^{q P} .
$$

Here $q P$ wave gets reflected and transmitted in case of normal incidence.

Case 2 (Quasitransverse ( $q S V$ ) wave incidence at an interface from semiconductor). We now consider the reflection of a plane $q S V$ wave for similar conditions on the boundary as in the previous section. For $q S V$ wave, we have

$$
\begin{aligned}
& \psi=\psi_{i}+\psi_{r} \\
& =A_{i} \exp \left\{\imath k_{3}(x \sin \theta-z \cos \theta)\right\} \\
& +A_{3} \exp \left\{\iota k_{3}\left(x \sin \theta_{3}+z \cos \theta_{3}\right)\right\} \text {, } \\
& \phi=\phi_{r}=\sum_{r=1}^{2} A_{r} \exp \left\{\iota k_{r}\left(x \sin \theta_{r}+z \cos \theta_{r}\right)\right\}, \\
& N=N_{r}=\sum_{r=1}^{2} S_{r} A_{r} \exp \left\{\iota k_{r}\left(x \sin \theta_{r}+z \cos \theta_{r}\right)\right\}, \\
& \phi_{L}=A_{4} \exp \left\{\iota k_{4}\left(x \sin \theta_{4}-z \cos \theta_{4}\right)\right\} \text {. }
\end{aligned}
$$

Upon using solution (46) in the boundary conditions (22) and (25) at the surface $z=0$ and assuming that all the incident or reflected waves are in phase at this surface for all values of $x$ and $t$, we have the relation

$$
k_{3} \sin \theta=k_{1} \sin \theta_{1}=k_{2} \sin \theta_{2}=k_{3} \sin \theta_{3} .
$$

This relation implies that

$$
\theta=\theta_{3}, \quad a_{1} \sin \theta_{1}=a_{2} \sin \theta_{2}=\frac{1}{\delta} \sin \theta_{3} .
$$

This is the modified form of Snell's law for the considered material, in this case. Upon solving (A.4) and (A.5), we get

$$
\begin{aligned}
R_{1}^{q S V} & =\frac{-S_{2} a_{2} \cos \theta_{2} \sin 4 \theta_{3}}{\bar{a} \Delta_{L}-\Delta_{S}}, \\
R_{2}^{q S V} & =\frac{a_{1} S_{1} \cos \theta_{1} \sin 4 \theta_{3}}{\bar{a} \Delta_{L}-\Delta_{S}}, \\
R_{3}^{q S V} & =\frac{\mathfrak{R}}{\left(\bar{a} \Delta_{L}-\Delta_{S}\right)}, \\
T_{1}^{q S V} & =\frac{2 \sin 2 \theta_{3} \Delta_{L}}{\bar{\rho} \Delta_{L}-a_{4} \delta \cos \theta_{4} \Delta_{S}} .
\end{aligned}
$$

where $\mathfrak{R}$ denotes $\left\{\bar{a} \Delta_{L}-\left\{a_{1} a_{2} \delta^{2}\left(a_{2} S_{1} \cos \theta_{1} \sin 2 \theta_{2}-a_{1} S_{2}\right.\right.\right.$ $\left.\left.\left.\cos \theta_{2} \sin 2 \theta_{1}\right) \times \sin 2 \theta_{3}-\left(a_{1} S_{1} \cos \theta_{1}-a_{2} S_{2} \cos \theta_{2}\right) \cos ^{2} 2 \theta_{3}\right\}\right\}$. 
Here $\Delta_{L}$ and $\Delta_{S}$ are defined in (40) and (41), respectively. In the absence of fluid medium (49) becomes

$$
\begin{aligned}
& R_{1}^{q S V}=\frac{S_{2} a_{2} \cos \theta_{2} \sin 4 \theta_{3}}{\Delta_{S}}, \\
& R_{2}^{q S V}=\frac{-a_{1} S_{1} \cos \theta_{1} \sin 4 \theta_{3}}{\Delta_{S}}, \\
& R_{3}^{q S V}=\frac{\mathcal{R}}{\Delta_{S}},
\end{aligned}
$$

where $\Delta_{S}$ is defined by (41). In the absence of fluid medium and electron field $\left(\varepsilon_{n}=0=N\right),(49)$ and (50) reduce to

$$
\begin{aligned}
& R_{1}^{q S V}= \frac{-\sin 4 \theta_{3}}{\cos ^{2} 2 \theta_{3}+\delta^{2} \sin 2 \theta_{1} \sin 2 \theta_{3}}, \\
& R_{2}^{q S V}=0, \\
& R_{3}^{q S V}=\frac{\delta^{2} \sin 2 \theta_{3} \sin 2 \theta_{1}-\cos ^{2} 2 \theta_{3}}{\cos ^{2} 2 \theta_{3}+\delta^{2} \sin 2 \theta_{3} \sin 2 \theta_{1}},
\end{aligned}
$$

Equations (51) agree with the corresponding equations in Achenbach [6] and Kino [7]. For grazing incidence $(\theta=$ $\left.90^{\circ}=\theta_{3}\right)$ and in case of normal incidence $\left(\theta=0^{\circ}=\theta_{3}\right)$, the expressions for reflection coefficients in (49)-(51) provide us

$$
R_{1}^{q S V}=0, \quad R_{2}^{q S V}=0, \quad T_{1}^{q S V}=0, \quad R_{3}^{q S V}=-1 .
$$

Therefore, only shear wave is reflected as $q S V$ wave in case of normal incidence, and the reflected $q S V$ wave annihilates the incident $q S V$ wave in case of grazing incidence case. The other two waves, namely, $q P$ and $N$, are not reflected, and there is no transmitted wave in either case of normal or grazing incidence.

\section{Energy Equations}

From the principle of conservation of energy, the energy carried to the boundary by the incident wave must be equal to the energy carried away from the boundary by the reflected and refracted waves. For the incident $q S V$ wave incidence the particle velocities are

$$
\begin{aligned}
\bar{v} & =\frac{\partial^{2} \psi_{i}}{\partial x \partial t} \\
& =\omega k_{3} \sin \theta A_{i} \exp \left\{\imath k_{3}(x \sin \theta-z \cos \theta-v t)\right\}, \\
\omega & =\frac{\partial^{2} \psi_{i}}{\partial z \partial t} \\
& =-\omega k_{3} \cos \theta_{3} A_{i} \exp \left\{i k_{3}(x \sin \theta-z \cos \theta-v t)\right\} .
\end{aligned}
$$

For reflected $q S V$ waves,

$$
\begin{aligned}
\bar{v} & =\frac{\partial^{2} \psi_{r}}{\partial x \partial t} \\
& =\omega k_{3} \sin \theta_{3} A_{3} \exp \left\{i k_{3}\left(x \sin \theta_{3}+z \cos \theta_{3}-v t\right)\right\} \\
\omega & =\frac{\partial^{2} \psi_{r}}{\partial z \partial t} \\
& =\omega k_{3} \cos \theta_{3} A_{3} \exp \left\{i k_{3}\left(x \sin \theta_{3}+z \cos \theta_{3}-v t\right)\right\}
\end{aligned}
$$

For reflected $q P$ waves,

$$
\begin{aligned}
\bar{v}= & \frac{\partial^{2} \phi_{r}}{\partial x \partial t} \\
= & \omega k_{1} \sin \theta_{1} A_{1} \exp \left\{i k_{1}\left(x \sin \theta_{1}+z \cos \theta_{1}-v t\right)\right\} \\
& +\omega k_{2} \sin \theta_{2} A_{2} \exp \left\{i k_{2}\left(x \sin \theta_{2}+z \cos \theta_{2}-v t\right)\right\}, \\
\omega= & \frac{\partial^{2} \phi_{r}}{\partial z \partial t} \\
= & \omega k_{1} \cos \theta_{1} A_{1} \exp \left\{i k_{1}\left(x \sin \theta_{1}+z \cos \theta_{1}-v t\right)\right\} \\
& +\omega k_{2} \cos \theta_{2} A_{2} \exp \left\{i k_{2}\left(x \sin \theta_{2}+z \cos \theta_{2}-v t\right)\right\} .
\end{aligned}
$$

For transmitted $q P$ waves,

$$
\begin{aligned}
\bar{v} & =\frac{\partial^{2} \phi_{L r}}{\partial x \partial t} \\
& =\omega k_{4} \sin \theta_{4} A_{4} \exp \left\{i k_{4}\left(x \sin \theta_{4}-z \cos \theta_{4}-v t\right)\right\}, \\
\omega & =\frac{\partial^{2} \phi_{L r}}{\partial z \partial t} \\
& =-\omega k_{4} \cos \theta_{4} A_{4} \exp \left\{i k_{4}\left(x \sin \theta_{4}-z \cos \theta_{4}-v t\right)\right\} .
\end{aligned}
$$

Taking the kinetic energy per unit volume as $(1 / 2) \rho\left(\bar{v}^{2}+\omega^{2}\right)$, we may calculate the energy flux for the waves mentioned above by multiplying the total energy per unit volume by the velocity of propagation and area of the wave front involved. Thus we may write the equality between the incident $q S V$ wave energy and the sum of reflected $q P$, reflected $q S V$, and transmitted $q S V$-wave energies for the per unit area on the interface as

$$
\begin{aligned}
c_{2} \cos \theta_{3} k_{3}^{2}= & c_{1} \cos \theta_{2} k_{1}^{2} R_{1}^{2}+c_{1} \cos \theta_{2} k_{2}^{2} R_{2}^{2} \\
& +c_{2} \cos \theta_{2} k_{3}^{2} R_{3}^{2}+\frac{\rho_{L}}{\rho} c_{L} \cos \theta_{4} k_{4}^{2} R_{4}^{2} .
\end{aligned}
$$

Here the cross-sectional areas of the incident, reflected, and transmitted waves are proportional to the cosines of the angles made by the ray directions of the waves with the normal to the interface. Hence the energy equation is given by

$$
1 \cong Z
$$

where

$$
Z=\frac{c_{2}}{c_{1}} \frac{\cos \theta_{2}}{\cos \theta_{3}} R_{1}^{2}+R_{3}^{2}-\frac{c_{2}}{c_{L}} \frac{\rho_{L}}{\rho} \frac{\cos \theta_{4}}{\cos \theta_{3}} R_{4}^{2}
$$

\section{7. (p-Type) Semiconductor}

The reflection coefficients and transmission coefficients in case of p-type semiconductor can be obtained by using boundary conditions (22)-(25) and solution (26) for the functions $\phi, \psi, \phi_{L}$, and $P$ by adopting the procedure of previous sections. The expressions of the reflection coefficients $R_{K}^{q P}$ and $R_{K}^{q S V}(k=1,2,3)$ are again given by (37)(42) and (49)-(51) in case of $q P$-wave, $q S V$-wave incident at the surface of p-type semiconductor, respectively, with the replacement of $a_{i}^{2}(i=1,2)$ as the $a_{i}^{* 2}(i=1,2)$ in all the relevant relations and equations including Snell's law. 
Table 1: Physical data of Silicon (Si) and Germanium (Ge) semiconductors.

\begin{tabular}{lccc}
\hline Coefficient & Unit & Value $(\mathrm{Ge})$ & Value $(\mathrm{Si})$ \\
\hline$\lambda$ & $\mathrm{Nm}^{-2}$ & $0.48 \times 10^{11}$ & $0.64 \times 10^{11}$ \\
$\mu$ & $\mathrm{Nm}^{-2}$ & $0.53 \times 10^{11}$ & $0.65 \times 10^{11}$ \\
$\rho$ & $\mathrm{Kgm}^{-3}$ & $5.3 \times 10^{3}$ & $2.3 \times 10^{3}$ \\
$t_{n}^{+}$ & $\mathrm{s}$ & $1 \mathrm{ps}$ & $1 \mathrm{ps}$ \\
$t_{p}^{+}$ & $\mathrm{s}$ & $1 \mathrm{ps}$ & $1 \mathrm{ps}$ \\
$D^{n}$ & $\mathrm{~m}^{2} \mathrm{~s}^{-1}$ & $10^{-2}$ & $0.35 \times 10^{-2}$ \\
$D^{p}$ & $\mathrm{~m}^{2} \mathrm{~s}^{-1}$ & $0.5 \times 10^{-2}$ & $0.125 \times 10^{-2}$ \\
$\alpha_{T}$ & $\mathrm{~K}^{-1}$ & $5.8 \times 10^{-6}$ & $2.6 \times 10^{-6}$ \\
$n_{0}=p_{0}$ & $\mathrm{~m}^{-3}$ & $10^{20}$ & $10^{20}$ \\
\hline
\end{tabular}

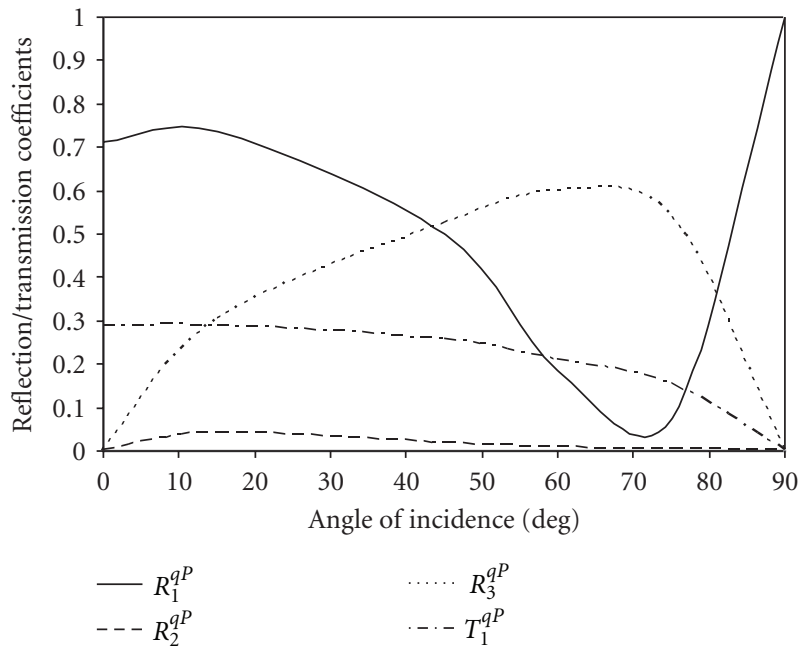

Figure 2: $q P$ wave incidence at the interface of semiconductor and fluid.

\section{Numerical Results and Discussion}

In this section the reflection and transmission coefficients given by (37)-(39) and (49)-(50) have been computed numerically for silicon $(\mathrm{Si})$ and germanium $(\mathrm{Ge})$ materials under the assumption of relaxation type semiconductor ( $\mathrm{n}$ type or p-type) under the assumption that semiconductor considered is of relaxation type so that $t_{n}, t_{n}^{+}$and $t_{p}, t_{p}^{+}$ become comparable to each other in their values such that $t_{n}=t_{n}^{+}$and $t_{p}=t_{p}^{+}$.

Here the fluid chosen for the purpose of numerical calculations is water, the velocity of sound in which is given by $c_{L}=1.5 \times 10^{3} \mathrm{~m} / \mathrm{s}$ and density is $\rho_{L}=1000 \mathrm{~kg} / \mathrm{m}^{3}$.

The physical data for silicon material is given below in Table 1 .

The values of reflection coefficients $R_{k}^{q P}(k=1,2,3)$, $R_{k}^{q S V}(k=1,2,3)$, and transmission coefficients $T_{1}^{q P}, T_{1}^{q S V}$ for incident $q P$ and $q S V$ waves have been computed from (37)-(39) and (49)-(50) for various values of the angle of incidence $(\theta)$ lying between $0^{\circ} \leq \theta \leq 90^{\circ}$ for silicon ( $\mathrm{Si}$ ) semiconductor.
From Figure 2, it is noticed that the magnitude of reflection coefficient $\left(R_{1}^{q P}\right)$ marginally increases in the range $0^{\circ} \leq \theta \leq 10^{\circ}$ with increase in angle of incidence and decreases in the range $10^{\circ} \leq \theta \leq 70^{\circ}$; that is, there is a sharp loss of energy which is noticed in the range $10^{\circ} \leq \theta \leq$ $70^{\circ}$ before it sharply increases upto $\theta=90^{\circ}$ in case of $q P$ wave incidence at the interface of semiconductor and fluid. The magnitude of reflection coefficient $\left(R_{3}^{q P}\right)$ increases for $0^{\circ} \leq \theta \leq 60^{\circ}$ and attains a maximum value at $\theta=75^{\circ}$; that is, loss of energy by reflected wave $\left(R_{1}^{q P}\right)$ is covered by reflected wave $\left(R_{3}^{q P}\right)$. However, a meager amount of energy is associated with an electron wave $\left(R_{2}^{q P}\right)$. It is also noticed that transmission coefficient $T_{1}^{q P}$ attains a maximum value at $\theta=0^{\circ}$ and then varies linearly up to $0^{\circ} \leq \theta \leq$ $15^{\circ}$; after that it starts decreasing up to $\theta=90^{\circ}$. This implies that in case of $q P$ wave incidence at the interface of semiconductor and fluid, transmitted wave also travels with sufficient amount of energy. It is revealed that at grazing incidence $\theta=90^{\circ}$, the reflection coefficients $R_{k}^{q P}(k=$ $1,2,3)$ and transmission coefficient $T_{1}^{q P}$ of incident $q P$ wave vanish, thereby meaning that reflected $q P$ wave annihilates the incident $q P$ wave. Whereas in contrast to this $q P$ wave is reflected and transmitted as $q P$ wave at normal incidence $\left(\theta=0^{\circ}\right)$, and other waves are not reflected or transmitted. Thus maximum energy is carried by longitudinal $(q P)$ waves, reflected or transmitted, at normal and grazing incidence, though it is transported in a distributed manner among all coupled waves at other angles of incidence. The trend and nature of reflection/transmission coefficients in case of $q P$ wave incidence in Figure 2 almost completely agree with those presented in Kino [7].

In Figure 3, it is noticed that reflection coefficient $R_{1}^{q S V}$ increases with increase in angle of incidence in the range $0^{\circ} \leq \theta \leq 30^{\circ}$ and attains a maximum value at $\left(\theta=30^{\circ}\right)$ due to stresses generated in the semiconductor material; after that it starts decreasing up to $30^{\circ} \leq \theta \leq 45^{\circ}$. Thus shear wave incidence has a critical angle at $\theta=45^{\circ}$. Beyond this cutoff point, the amplitude of longitudinal wave component at the surface is finite, but no real power is associated with them; only decaying fields are associated with them. However, the reflection coefficient $R_{2}^{q S V}$ increases sharply and attains a maximum energy at $\theta=30^{\circ}$, then decreases for 


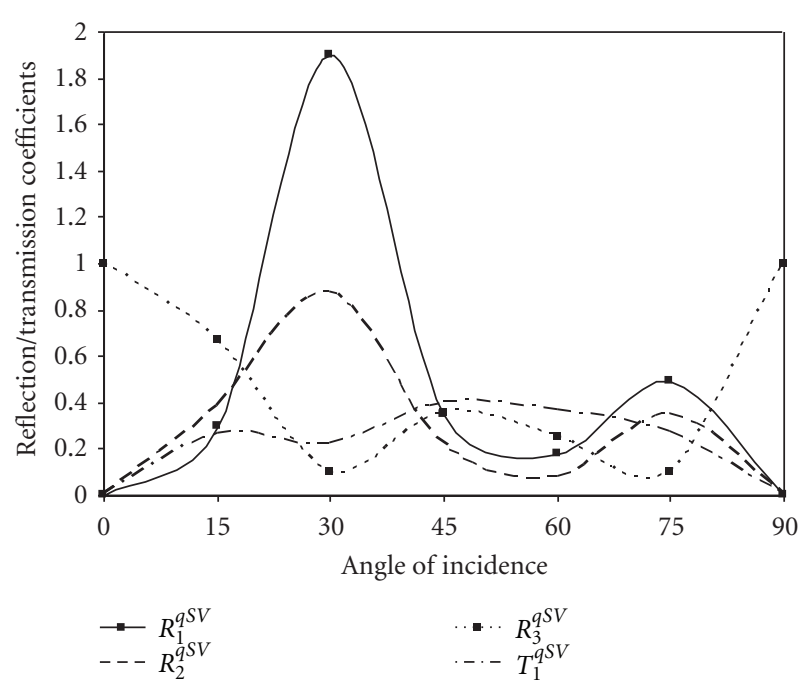

FIGURE 3: $q S V$ wave incidence at the interface of semiconductor and fluid.

TABLE 2: Reflection/transmission coefficients in Germanium (Ge) semiconductor.

\begin{tabular}{lcccc}
\hline \multirow{2}{*}{ Angle of incidence $(\theta)$} & \multicolumn{4}{c}{ Reflection/transmission coefficients } \\
& $R_{1}^{q S V}$ & $R_{2}^{q S V}$ & $R_{3}^{q S V}$ & $T_{1}^{q S V}$ \\
\hline $0^{\circ}$ & 0 & 0 & 1 & 0 \\
$15^{\circ}$ & 0.4966 & 0.0538 & 0.0281 & 0.1848 \\
$30^{\circ}$ & 0.2522 & 0.9208 & 0.1210 & 0.4863 \\
$45^{\circ}$ & 0.2023 & 0.01313 & 0.8723 & 0.5759 \\
$60^{\circ}$ & 0.0653 & 0.0006 & 0.9326 & 0.6391 \\
$75^{\circ}$ & 0.2939 & 0.2980 & 0.5814 & 0.7313 \\
$90^{\circ}$ & 0 & 0.0759 & 1 & 0.1094 \\
\hline
\end{tabular}

$30^{\circ} \leq \theta \leq 60^{\circ}$; this means that electron wave gets sufficient amount of energy before it dies out at $\theta=90^{\circ}$ which is observed to be a new phenomenon here. The reflection coefficient $R_{3}^{q S V}$ for $q S V$ wave decreases in the range $0^{\circ} \leq$ $\theta \leq 30^{\circ}$, increases up to $\theta=45^{\circ}$ and remains steady for $45^{\circ} \leq \theta \leq 60^{\circ}$ and again decreases to attain its minimum value at $\theta=72^{\circ}$, and then sharply increases up to $\theta=$ $90^{\circ}$; that is, it recovers from the initial loss of energy. $T_{1}^{q S V}$ increases in the range $0^{\circ} \leq \theta \leq 30^{\circ}$ to attain its maximum value at $\theta=45^{\circ}$ and decreases up to $\theta=90^{\circ}$. It is also noticed that about $40 \%$ of incident energy can be converted to a longitudinal wave in water at incident angles for which the reflected longitudinal wave is cutoff. Thus solid-liquid interface is a perfect reflector.

Figure 4, the variation of reflection coefficients $R_{k}^{q S V}(k=$ $1,3)$, and transmission coefficients $T_{1}^{q S V}$ versus angle of incidence have been plotted. It is noticed that the reflection coefficient $R_{1}^{q S V}$ increases sharply with increase in the angle of incidence and attains a significantly large value at $\theta=30^{\circ}$ and after that it starts decreasing up to a critical angle $\theta=$ $45^{\circ}$, which is $\theta=36^{\circ}$ in case of stress-free boundary. This is attributed due to high stress generation in the material at this angle of incidence. Beyond this cutoff point whole of

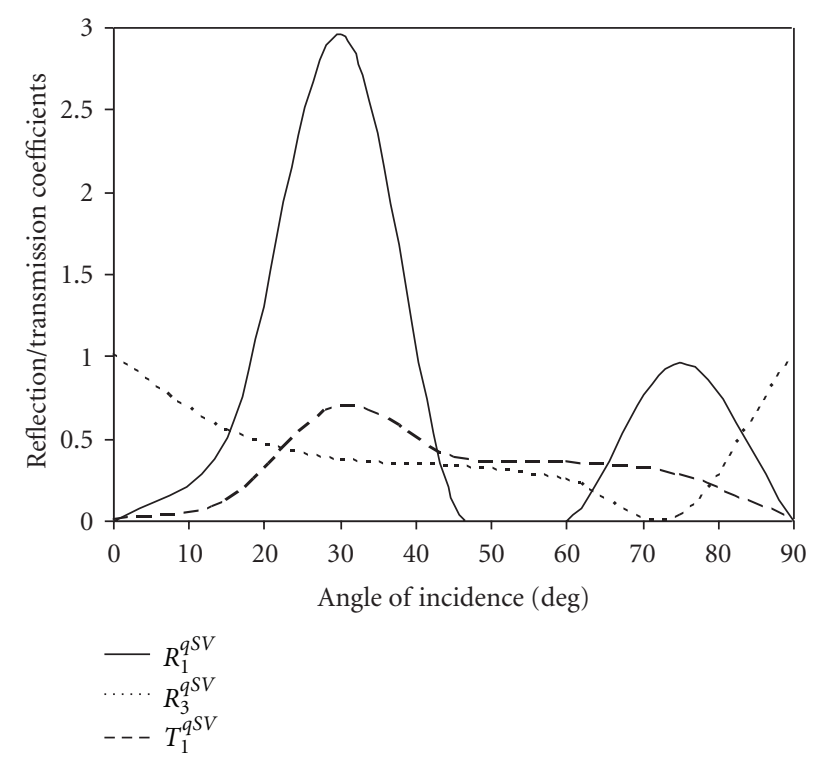

FIGURE 4: $q S V$ wave incidence at the interface of semiconductor and fluid under electron field equilibrium.

the incident shear wave power is converted into a reflected $q S V$ wave so that $\left|R_{3}^{q S V}\right|=1$ approximately, although there is a $\pi$-phase shift of $R_{3}^{q S V}$ as $\theta$ passes through the critical angle in case of $q S V$ wave incident at the stressfree, isoconcentrated, or impermeable surface of silicon (Si) half-space [20]. Transmission coefficient $T_{1}^{q S V}$ increases with increase in angle of incidence and attains a maximum value at $\theta=30^{\circ}$; after that it starts decreasing and vanishes at $\theta=90^{\circ}$. This implies that major portion of energy is carried by transmitted wave in comparison to $R_{3}^{q S V}$ in $q S V$ wave incidence at the interface of semiconductor and fluid under electron field equilibrium.

From Table 2 it is noticed that the behaviors of reflection/transmission coefficients of various waves in germanium $(\mathrm{Ge})$ semiconductor halfspace are almost similar to that in case of silicon $(\mathrm{Si})$ semiconductor except some minor changes in their magnitudes. From Table 3, we concluded that the law of conservation of energy is valid.

\section{Conclusions}

It is noticed that the magnitude of reflection coefficient $\left(R_{1}^{q P}\right)$ of $q P$ wave decreases with increasing angle of incidence of $q P$ wave in case of silicon $(\mathrm{Si})$ semiconductor material halfspace in the range $0^{\circ} \leq \theta \leq 70^{\circ}$, and the numerical results show that in case of $q P$ wave incidence maximum energy is carried by transmitted longitudinal wave in the presence of electron wave which takes meager amount of energy. Thus energy transfer is by the phonon of the system, and partition of energy depends upon the angle of incidence. However in case of $q S V$ wave incidence at the surface, transmitted wave becomes more prominent in the presence of electron field and energy is transported in distributed manner among the other waves. The dependence of $R_{i}^{q P}(i=1,2,3)$ on $a_{i}(1,2)$ 
TABLE 3: Variation of energy coefficients $(Z)$ in case of $(q S V)$ wave incidence versus angle of incidence $(\theta)$ in degree.

\begin{tabular}{lccccccc}
\hline Angle of incidence $(\theta)$ in degree & 0 & 15 & 30 & 45 & 60 & 75 & 90 \\
\hline Energy coefficients $(Z)$ & 0.8720 & 1.3524 & 2.9889 & 0.99417 & 0.0437 & 1.1923 & 0.2224 \\
\hline
\end{tabular}

shows that the reflection coefficients do depend upon the frequency of waves and hence are dispersive in character. The study may find application in semiconductor, seismology, and signal processing devices in coated structures.

\section{Appendix}

Upon employing the boundary conditions (22) and (25) following system of equations at the surface $(z=0)$ for the n-type semiconductor is obtained

$$
\begin{aligned}
& \left(2 \delta^{2} k_{1}^{2} \sin ^{2} \theta-\omega^{2}\right) A_{i} e^{\iota k_{1}(x \sin \theta)} \\
& +\sum_{j=1}^{2}\left(2 \delta^{2} k_{j}^{2} \sin ^{2} \theta_{j}-\omega^{2}\right) A_{j} e^{l k_{j}\left(x \sin \theta_{j}\right)} \\
& +\delta^{2} k_{3}^{2} \sin 2 \theta_{3} A_{3} e^{\imath k_{3}\left(x \sin \theta_{3}\right)}=-\bar{\rho} \omega^{2} A_{4} e^{\imath k_{4} x \sin \theta_{4}}, \\
& \left(\delta^{2} k_{1}^{2} \sin 2 \theta\right) A_{i} e^{\imath k(x \sin \theta)} \\
& +\sum_{j=1}^{2}\left(\delta^{2} k_{j}^{2} \sin 2 \theta_{j}\right) A_{1} e^{\imath k_{j}\left(x \sin \theta_{j}\right)} \\
& +\left(2 \delta^{2} k_{3}^{2} \sin ^{2} \theta_{3}-\omega^{2}\right) A_{3} e^{\imath k_{3}\left(x \sin \theta_{3}\right)}=0 \\
& -\imath k_{1} \cos \theta A_{i} e^{\imath k_{1}(x \sin \theta)}+\sum_{j=1}^{2} \iota k_{j} \cos \theta_{j} A_{j} e^{\imath k_{j}\left(x \sin \theta_{j}\right)} \\
& -\imath k_{3} \sin \theta_{3} A_{3} e^{\imath k_{3}\left(x \sin \theta_{3}\right)}+\imath k_{4} \cos \theta_{4} A_{4} e^{\imath k_{4}\left(x \sin \theta_{3}\right)} \\
& -S_{1} k_{1} \cos \theta_{1} A_{i} e^{\imath k(x \sin \theta)} \\
& +\sum_{j=1}^{2} S_{j} k_{j} \cos \theta_{j} A_{j} e^{\imath k_{j}\left(x \sin \theta_{j}\right)}=0
\end{aligned}
$$

The system of (A.1) with the help of (35) becomes

$$
A Z_{P}=C_{1},
$$

where the matrices $A$ and $C_{1}$ are given by

$$
A=\left[\begin{array}{cccc}
\cos 2 \theta_{3} & \cos 2 \theta_{3} & -\sin 2 \theta_{3} & -\bar{\rho} \\
a_{1}^{2} \delta^{2} \sin 2 \theta_{1} & a_{2}^{2} \delta^{2} \sin 2 \theta_{2} & \cos 2 \theta_{3} & 0 \\
a_{1} \delta \cos \theta_{1} & a_{2} \delta \cos \theta_{2} & -\sin \theta_{3} & a_{4} \delta \cos \theta_{4} \\
a_{1} S_{1} \cos \theta_{1} & a_{2} S_{2} \cos \theta_{2} & 0 & 0
\end{array}\right]
$$

$$
\begin{aligned}
& Z_{P}=\left[R_{1}^{q P}, R_{2}^{q P}, R_{3}^{q P}, T_{1}^{q P}\right]^{T}, \\
& C_{1}=\left[-\cos 2 \theta_{3}, a_{1}^{2} \delta^{2} \sin 2 \theta_{1}, a \delta \cos \theta_{1}, a_{1} S_{1} \cos \theta_{1}\right]^{T} .
\end{aligned}
$$

Here $R_{k}^{q P}=A_{k} / A_{i},(k=1,2,3)$ are amplitude ratios of the reflected waves to the incident waves, and $T_{1}^{q P}=A_{4} / A_{i}$ are the amplitude ratios of transmitted wave to the incident wave. Upon applying the appropriate boundary conditions prevailing at the surface of the semiconductor half-space, the amplitude ratios of $q S V$-wave reflection are given by the matrix equations as

$$
A Z_{S V}=C_{2}
$$

where the matrices $Z_{S V}$ and $C_{2}$ are given by

$$
\begin{gathered}
Z_{S V}=\left[R_{1}^{q S V}, R_{2}^{q S V}, R_{3}^{q S V}, T_{1}^{q S V}\right]^{T}, \\
C_{2}=\left[-\sin 2 \theta_{3},-\cos 2 \theta_{3}, \sin \theta_{3}, 0\right]^{T}
\end{gathered}
$$

and the matrices $A$ is defined in (A.3).

\section{References}

[1] H. Jeffrey's, "The thermodynamics of elastic solids," Proceeding of the Cambridge Philosophical Society, vol. 26, pp. 101-106, 1930.

[2] B. Gutenberg, "Relation of reflected and refracted seismic waves," Bulletin of the Seismological Society of America, vol. 34, pp. 85-102, 1944.

[3] R. S. Sidhu and S. J. Singh, "Reflection of P and SV waves at the free surface of a prestressed elastic half-space," Journal of Acoustical Society of America, vol. 76, no. 2, pp. 594-598, 1984.

[4] L. Rayleigh, "On the dynamical theory of grating," Proceeding of the Royal Society of London Series A, vol. 79, pp. 399-416, 1907.

[5] C.G. Knott, "Reflection and refraction of elastics waves with seismological Applications," Philosophical Magazine, vol. 48, pp. 64-97, 1899.

[6] J. D. Achenbach, Wave Propagation in Elastic Solids, North Holland Publishing Company, Amsterdam, The Netherlands, 1973.

[7] G. S. Kino, Acoustic Waves: Devices Imaging and Analog Signal Processing, Prentice Hall, Englewood Cliffs, NJ, USA, 1987.

[8] K. F. Graff, Wave Motion in Elastic Solids, Dover, New York, NY, USA, 1991.

[9] H. Deresiewicz, "Effect of boundaries on waves in a thermoelastic solid: reflexion of plane waves from a plane boundary," Journal of the Mechanics and Physics of Solids, vol. 8, no. 3, pp. $164-185,1960$.

[10] S. M. Abo-Dahab, "Propagation of $P$ waves from stress-free surface elastic half-space with voids under thermal relaxation and magnetic field," Applied Mathematical Modelling, vol. 34, no. 7, pp. 1798-1806, 2010.

[11] A. Madeo and S. Gavrilyuk, "Propagation of acoustic waves in porous media and their reflection and transmission at a purefluid/porous-medium permeable interface," European Journal of Mechanics, A/Solids, vol. 29, no. 5, pp. 897-910, 2010.

[12] A. N. Sinha and S. B. Sinha, "Reflection of thermoelastic waves at a solid half-space with thermal relaxation," Journal of Physics of the Earth, vol. 22, no. 2, pp. 237-244, 1974.

[13] J. N. Sharma, V. Kumar, and D. Chand, "Reflection of generalized thermoelastic waves from the boundary of a halfspace," Journal of Thermal Stresses, vol. 26, no. 10, pp. 925-942, 2003. 
[14] A. N. Abd-alla, "Relaxation effects on reflection of generalized magneto-thermoelastic waves," Mechanics Research Communications, vol. 27, no. 5, pp. 591-600, 2000.

[15] F. J. Lockett, "Effect of thermal properties of a solid on the velocity of Rayleigh waves," Journal of the Mechanics and Physics of Solids, vol. 7, no. 1, pp. 71-75, 1958.

[16] P. Chadwick and I. N. Snedon, "Plane waves in an elastic solid conducting heat," Journal of the Mechanics and Physics of Solids, vol. 6, no. 3, pp. 223-230, 1958.

[17] B. Maruszewski, "Thermo- diffusive surface waves in semiconductors," Journal of Acoustic Society of America, vol. 85, pp. 1967-1977, 1989.

[18] J. N. Sharma and N. Thakur, "Plane harmonic elasto-thermodiffusive waves in semiconductor materials," Journal of Mechanics of Materials and Structure, vol. 1, pp. 813-835, 2006.

[19] J. N. Sharma and A. Sharma, "Reflection of acoustodiffusive waves from the boundary of a semiconductor halfspace," Journal of Applied Physics, vol. 108, no. 3, pp. 033712-033723, 2010.

[20] J. N. Sharma, I. Sharma, and S. Chand, "Elasto-thermodiffusive surface waves in a semiconductor half-space underlying a fluid with varying temperature," Journal of Thermal Stresses, vol. 31, no. 10, pp. 956-975, 2008.

[21] S. M. Sze, Physics of Semiconductor Devices, John Wiley \& Sons, New York, NY, USA, 1981.

[22] M. Zambuto, Semiconductor Devices, McGraw-Hills, New York, NY, USA, 1989. 

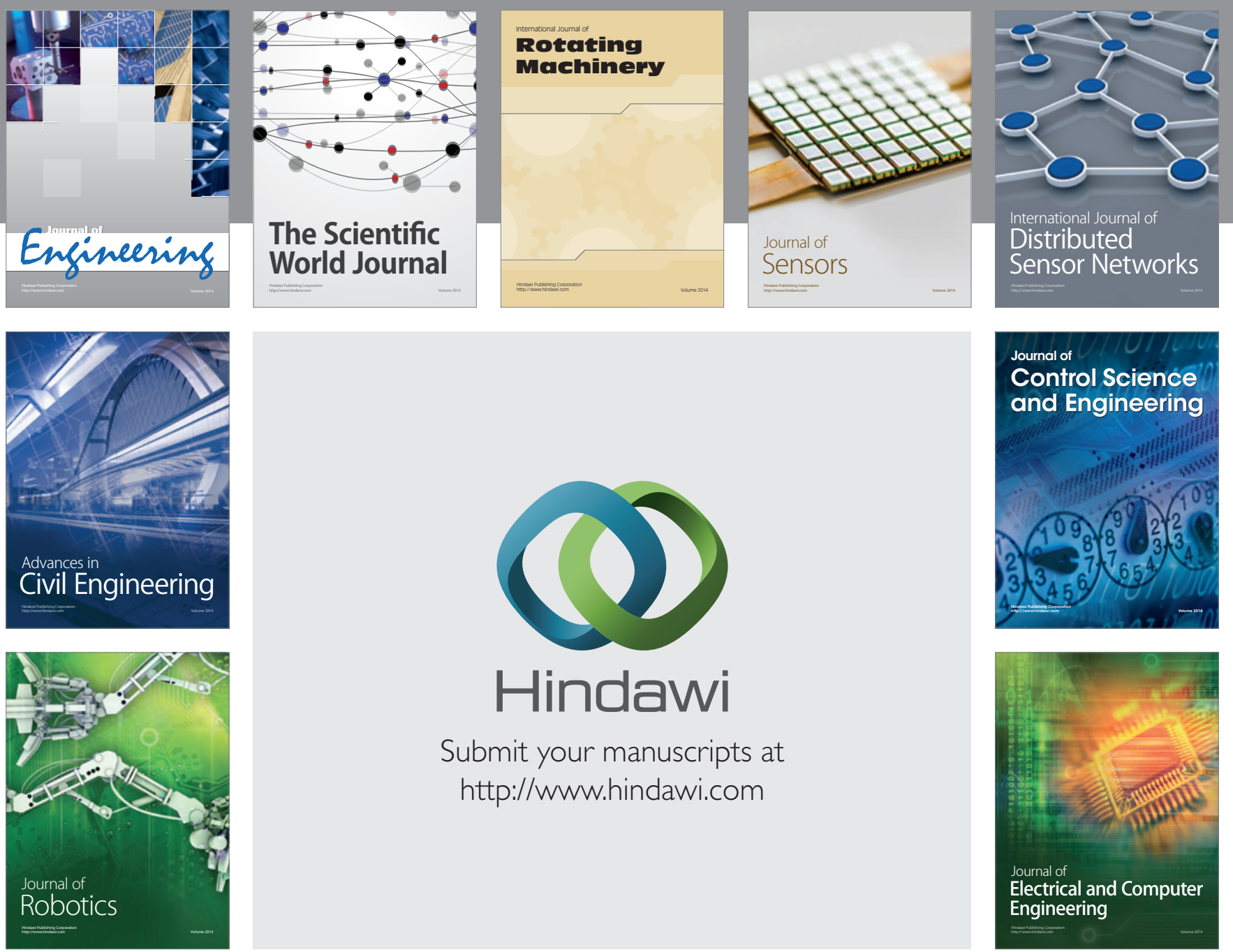

Submit your manuscripts at

http://www.hindawi.com
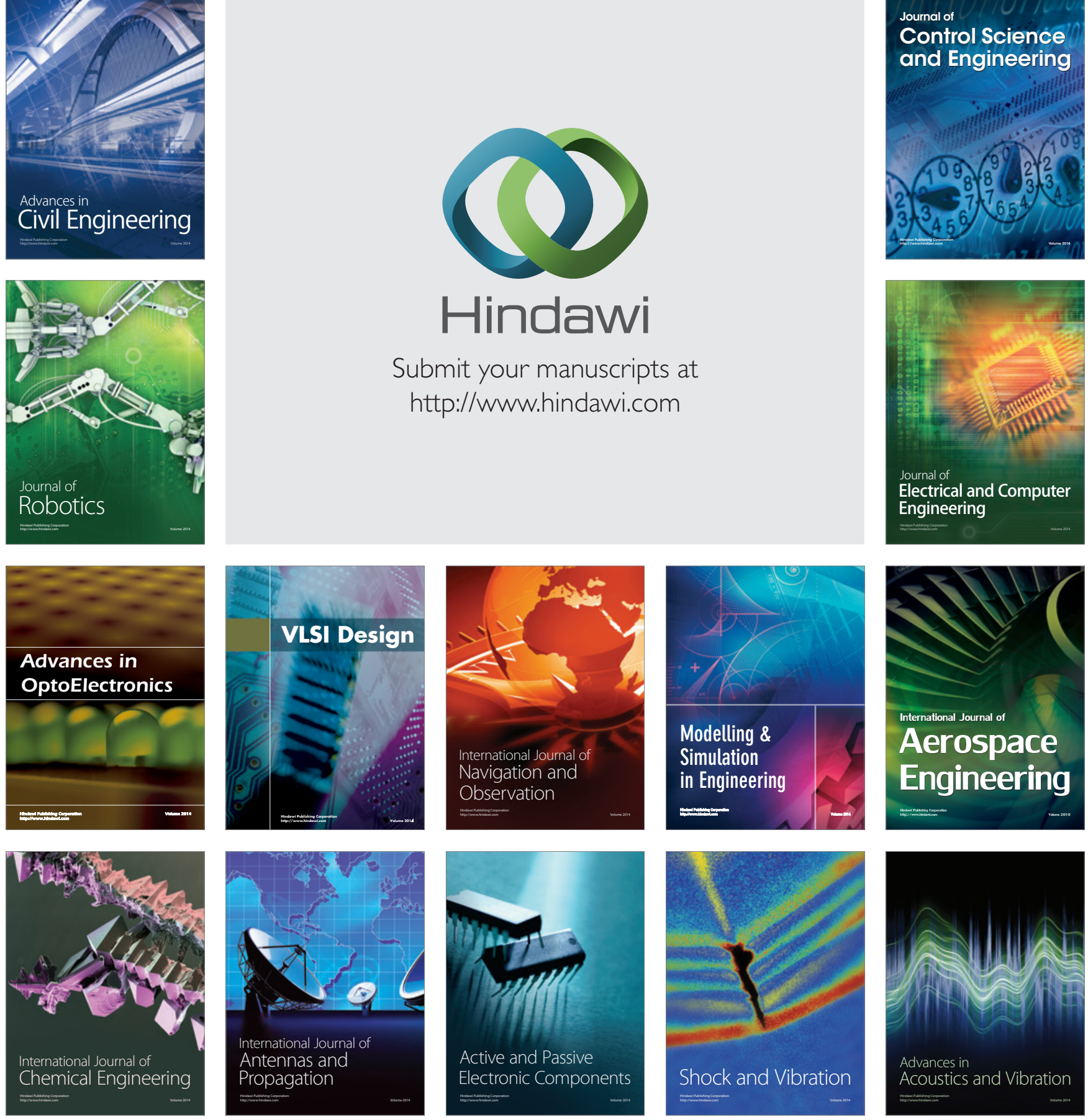